\title{
Correction to: Depth functions of symbolic powers of homogeneous ideals
}

\section{Hop Dang Nguyen ${ }^{1}$ • Ngo Viet Trung ${ }^{2}$}

Received: 20 August 2019 / Accepted: 9 September 2019 /

Published online: 11 October 2019

(C) Springer-Verlag GmbH Germany, part of Springer Nature 2019

\section{Correction to: Invent. math.} https://doi.org/10.1007/s00222-019-00897-y

The original proof of Theorem 3.3 is incorrectly claims that $\overline{I^{(t)}}=$ $\bigcap_{F \in \mathcal{F}(I)} \overline{I_{F}^{t}}$. We have found a counter-example to this claim. The proof remains correct if we replace $\overline{I^{(t)}}$ by $\bigcap_{F \in \mathcal{F}(I)} \overline{I_{F}^{t}}$ for all $t \geq 1$; see the corrected proof below. The correction concerns only this proof and does not affect any result of the paper.

Theorem 3.3 Let I be a monomial ideal in $R$ such that $I^{(t)}$ is integrally closed for $t \gg 0$. Then depth $R / I^{(t)}$ is a convergent function with

$$
\lim _{t \rightarrow \infty} \operatorname{depth} R / I^{(t)}=\operatorname{dim} R-\operatorname{dim} F_{S}(I),
$$

The original article can be found online at https://doi.org/10.1007/s00222-019-00897-y.

Ngo Viet Trung

nvtrung@math.ac.vn

Hop Dang Nguyen

ngdhop@gmail.com

1 Institute of Mathematics, Vietnam Academy of Science and Technology, 18 Hoang Quoc Viet, Hanoi, Vietnam

2 International Centre for Research and Postgraduate Training, Institute of Mathematics, Vietnam Academy of Science and Technology, 18 Hoang Quoc Viet, Hanoi, Vietnam 
which is also the minimum of depth $R / I^{(t)}$ among all integrally closed symbolic powers $I^{(t)}$.

Proof Let $m$ be the minimum of depth $R / I^{(t)}$ among all integrally closed symbolic powers $I^{(t)}$. Choose an integrally closed symbolic power $I^{(s)}$ such that depth $R / I^{(s)}=m$. By Theorem 2.6(ii), there exists an integer $a$ such that depth $R / I^{(s)} \geq \operatorname{depth} R / I^{(t)}$ for $t \geq a s^{2}$. This implies depth $R / I^{(t)}=m$ for all integrally closed symbolic powers $I^{(t)}$ with $t \geq a s^{2}$. Since $I^{(t)}$ is integrally closed for $t \gg 0$, we get depth $R / I^{(t)}=m$ for $t \gg 0$.

Let $I_{t}=\bigcap_{F \in \mathcal{F}(I)} \overline{I_{F}^{t}}$. We will show that $m=\min _{t \geq 1}$ depth $R / I_{t}$. Since $I_{t}=I^{(t)}$ for $t \gg 0$ by Proposition 2.2,

$$
m \geq \min _{t \geq 1} \operatorname{depth} R / I_{t}
$$

By Proposition 2.3, we have

$$
\operatorname{depth} R / I_{s} \geq \operatorname{depth} R /\left(I_{S}\right)^{(t)}
$$

for all $s, t \geq 1$. For $t \gg 0, I^{(s t)}$ is integrally closed and so is $I_{F}^{s t}$ for all $F \in \mathcal{F}(I)$ by Proposition 2.2. This implies $I_{F}^{s t} \subseteq\left(\overline{I_{F}^{s}}\right)^{t} \subseteq \overline{I_{F}^{s t}}=I_{F}^{s t}$. Hence, $\left(\overline{I_{F}^{s}}\right)^{t}=I_{F}^{s t}$. So we get

$$
\left(I_{S}\right)^{(t)}=\bigcap_{F \in \mathcal{F}(I)} I_{F}^{s t}=I^{(s t)}
$$

Therefore,

$$
\operatorname{depth} R / I_{s} \geq \operatorname{depth} R / I^{(s t)} \geq m
$$

for all $s \geq 1$. Now, we can conclude that

$$
m=\min _{t \geq 1} \operatorname{depth} R / I_{t}
$$

It remains to show that $\min _{t \geq 1} \operatorname{depth} R / I_{t}=\operatorname{dim} R-\operatorname{dim} F_{S}(I)$. For that, we need the following auxiliary observation (cf. [41, Proposition 2.5]).

Let $\mathcal{F}$ denote the filtration of the ideals $I_{t}, t \geq 0$. Let $R(\mathcal{F})=\bigoplus_{t \geq 0} I_{t} y^{t}$. Then $R(\mathcal{F})$ is an algebra generated by monomials in $k\left[x_{1}, \ldots, x_{n}, y\right]$. We have

$$
R(\mathcal{F})=\bigcap_{F \in \mathcal{F}(I)} \bigoplus_{t \geq 0} \overline{I_{F}^{t}} y^{t} .
$$


For each $F \in \mathcal{F}(I)$, the algebra $\bigoplus_{t \geq 0} \overline{I_{F}^{t}} y^{t}$ is the normalization of the finitely generated algebra $\bigoplus_{t \geq 0} I_{F}^{t} y^{t}$. Hence, $\bigoplus_{t \geq 0} \overline{I_{F}^{t}} y^{t}$ is a finitely generated algebra. The monomials of $\bigoplus_{t \geq 0} \overline{I_{F}^{t}} y^{t}$ form a finitely generated semigroup. Since the semigroup of the monomials of $R(\mathcal{F})$ is the intersections of these semigroups, it is also finitely generated [14, Corollary 1.2]. From this, it follows that $R(\mathcal{F})$ is a finitely generated algebra. Moreover, as an intersection of normal rings, $R(\mathcal{F})$ is a normal ring. By [20, Theorem 1], this implies that $R(\mathcal{F})$ is Cohen-Macaulay.

Let $G(\mathcal{F})=\bigoplus_{t \geq 0} I_{t} / I_{t+1}$. Then $G(\mathcal{F})$ is a factor ring of $R(\mathcal{F})$ by the ideal $\bigoplus_{t>0} I_{t+1} y^{t}$. Hence, $G(\mathcal{F})$ is a finitely generated algebra. By [4, Theorem 4.5.6(b)], we have $\operatorname{dim} G(\mathcal{F})=\operatorname{dim} R$. By the proof of the necessary part of [40, Theorem 1.1], the Cohen-Macaulayness of $R(\mathcal{F})$ implies that of $G(\mathcal{F})$. By [5, Theorem 9.23], these facts imply

$$
\begin{aligned}
\min _{t \geq 1} \operatorname{depth} R / I_{t} & =\operatorname{grade} \mathfrak{m} G(\mathcal{F})=\mathrm{ht} \mathfrak{m} G(\mathcal{F}) \\
& =\operatorname{dim} G(\mathcal{F})-\operatorname{dim} G(\mathcal{F}) / \mathfrak{m} G(\mathcal{F}) \\
& =\operatorname{dim} R-\operatorname{dim} G(\mathcal{F}) / \mathfrak{m} G(\mathcal{F}) .
\end{aligned}
$$

We have $G(\mathcal{F}) / \mathfrak{m} G(\mathcal{F})=\bigoplus_{t \geq 0} I_{t} /\left(\mathfrak{m} I_{t}+I_{t+1}\right)$. Since $F_{s}(I)=\bigoplus_{t \geq 0} I^{(t)} /$ $\mathfrak{m} I^{(t)}, I^{(t+1)} \subseteq \mathfrak{m} I^{(t)}$ [10, Proposition 9] and $I_{t}=I^{(t)}$ for $t \gg 0$, the graded algebras $G(\mathcal{F}) / \mathfrak{m} G(\mathcal{F})$ and $F_{S}(I)$ share the same Hilbert quasi-polynomial [4, Theorem 4.4.3]. From this, it follows that $\operatorname{dim} G(\mathcal{F}) / \mathfrak{m} G(\mathcal{F})=\operatorname{dim} F_{S}(I)$. Therefore,

$$
\min _{t \geq 1} \operatorname{depth} R / I_{t}=\operatorname{dim} R-\operatorname{dim} F_{S}(I) \text {. }
$$

Moreover, the reference [40] lists the wrong year. It has to be 1989 instead of 1997.

Acknowledgements The original paper and this correction are supported by Vietnam National Foundation for Science and Technology Development under Grant Number 101.04-2019.313. The authors thank Arvind Kumar for pointing out the mistake of the original proof.

Publisher's Note Springer Nature remains neutral with regard to jurisdictional claims in published maps and institutional affiliations. 